\title{
Early gastric adenocarcinoma of the fundic gland type
}
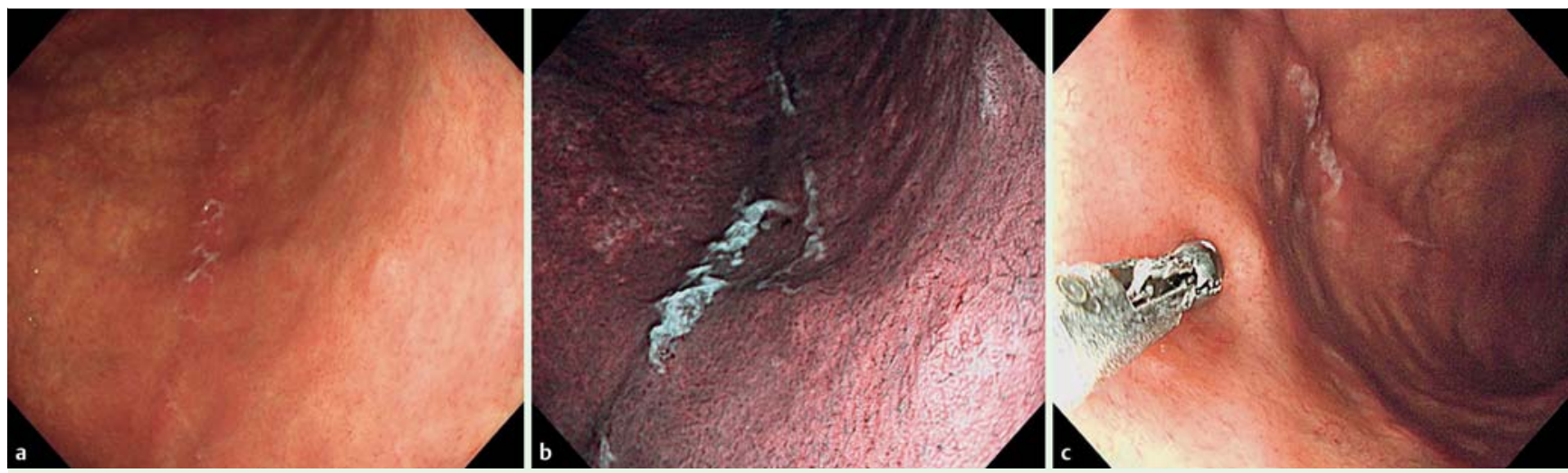

Fig. 1 Endoscopic views of a small polypoid lesion that was found to be a gastric adenocarcinoma of fundic gland type: a on white-light imaging; b on narrowband imaging; $\mathbf{c}$ as the whitish lesion is biopsied.

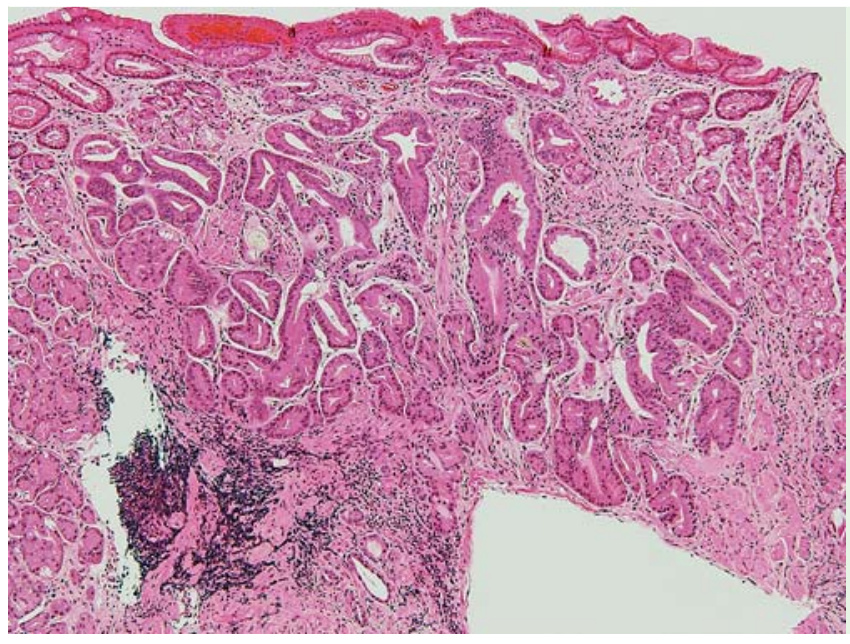

Fig. 2 Histology of the hematoxylin and eosin (H\&E)-stained biopsy specimen showing gastric adenocarcinoma of the fundic gland type (original magnification $\times 10$ ).
An asymptomatic 79-year-old man had been undergoing esophagogastroduodenoscopy annually since having an endoscopic resection of an intramucosal welldifferentiated gastric adenocarcinoma of the middle body 9 years previously. He had also received Helicobacter pylori eradication therapy, which had been successful, 10 years previously.

On this occasion, his follow-up esophagogastroduodenoscopy revealed a minute submucosal tumor-like polypoid lesion with some whitish discoloration on the greater curvature of the gastric lower body ( $\bullet$ Fig.1a). Narrow-band imaging showed non-neoplastic pits that were slightly dilated ( Fig.1b). Complete resection was attempted using a deep biopsy procedure because a carcinoid tumor was suspected and the size of the lesion was small (approximately $1.6 \mathrm{~mm}$ in diameter by comparison to the forceps) ( $\bullet$ Fig. 1 c). Histological examination of the biopsy specimen revealed atypical tubules made up of cells with basophilic cytoplasm ( $\bullet$ Fig. 2). These were covered with a non-neoplastic epithelium and were invading the muscularis mucosae with fibromusculosis. No lymphovascular invasion was observed. Immunohistochemical analysis revealed that these tubules were diffusely positive for pepsinogen I and
MUC6, positive for $\mathrm{H}+/ \mathrm{K}+$ ATPase in scattered locations around the tumor margin, and negative for MUC2, MUC5AC, CD10, and chromogranin $\mathrm{A}$.

The patient was diagnosed with gastric adenocarcinoma of the fundic gland, chief cell predominant type. Endoscopic submucosal dissection was performed, but no remnants of the tumor were present. No evidence of metastasis was observed on a computed tomography (CT) scan 1 year later.

Gastric adenocarcinoma, fundic gland type (GA-FG) has recently been proposed as a new entity of gastric adenocarcinoma [1]. GA-FG chief cell predominant types (GA-FG-CCPs) mostly develop without Helicobacter pylori infection [2] and often invade the submucosa independently of size $[1,2]$.

Endoscopically, early GA-FG-CCPs may mimic early carcinoid tumors because both of these tumors are histologically centered in the deep mucosa ( $\bullet$ Fig. 2) [3]. It has been reported on endoscopic evaluation that half of GA-FG-CCPs have a submucosal tumor shape with a whitish color [2]. Our case suggests that the early features of GA-FG-CCP include the above characteristics.

Endoscopy_UCTN_Code_CCL_1AB_2AD_3AB

\section{Competing interests: None}




\section{Kazutoshi Hori ${ }^{1}$, Yoshi-Hiro Ide ${ }^{2}$, Seiichi Hirota ${ }^{2}$, Fumihiko Toyoshima ${ }^{3}$, Tetsuya Takagawa ${ }^{1}$, Shiro Nakamura ${ }^{1}$}

${ }^{1}$ Department of Intestinal Inflammation Research, Hyogo College of Medicine, Nishinomiya, Hyogo, Japan

2 Department of Surgical Pathology, Hyogo College of Medicine, Nishinomiya, Hyogo, Japan

${ }^{3}$ Division of Gastroenterology, Department of Internal Medicine, Hyogo College of Medicine, Nishinomiya, Hyogo, Japan

\section{References}

1 Ueyama H, Yao T, Nakashima Y et al. Gastric adenocarcinoma of fundic gland type (chief cell predominant type): proposal for a new entity of gastric adenocarcinoma. Am J Surg Pathol 2010; 34: 609-619

2 Ueyama H, Matsumoto K, Nagahara A et al. Gastric adenocarcinoma of the fundic gland type (chief cell predominant type). Endoscopy 2014; 46: $153-157$

3 Singhi AD, Lazenby AJ, Montgomery EA. Gastric adenocarcinoma with chief cell differentiation: a proposal for reclassification as oxyntic gland polyp/adenoma. Am J Surg Pathol 2012; 36: 1030-1035

\section{Bibliography}

DoI http://dx.doi.org/

10.1055/s-0034-1391500

Endoscopy 2015; 47: E177-E178

(c) Georg Thieme Verlag KG

Stuttgart · New York

ISSN 0013-726X

\section{Corresponding author}

\section{Kazutoshi Hori, MD, PhD}

Department of Intestinal Inflammation Research Hyogo College of Medicine

1-1 Mukogawa-cho

Nishinomiya 663-8501

Japan

Phone: +81-798-456660

k-hori@hyo-med.ac.jp 